\title{
Mutualisation des informations en réunion de relève infirmière: structures préférentielles, structures dédiées
}

\section{Mutualising information in nurses' handovers: Preferential structures, dedicated structures}

\author{
Louis Maritaud $^{1}$ et Sandra Teston-Bonnard ${ }^{1}$ \\ ${ }^{1}$ Laboratoire ICAR UMR 5191 (CNRS, ENS de Lyon, Université Lumière Lyon 2), France
}

\begin{abstract}
Résumé. Les réunions de relève infirmière en psychiatrie sont des moments d'échanges importants dans la pratique professionnelle des soignants. Ces situations de mutualisation d'informations sont couplées à l'existence de notes écrites, créant ainsi deux modalités de transmission d'informations. Cet article propose d'établir, à partir des outils de la macrosyntaxe Aixoise et de l'analyse conversationnelle, des structures appuyant le discours des soignants. Nous abordons, à partir de l'analyse de relèves écrites et orales concernant une patiente, des phénomènes syntaxiques, lexicaux et pragmatiques qui nous permettent de distinguer les objectifs des deux modalités de relève, ainsi que leurs réalisations. Puis, nous analysons un phénomène transversal aux productions écrites et orales, le discours rapporté, au travers d'une observation écrite correspondant aux informations mutualisées lors d'une relève orale. Forts de ces analyses, nous pouvons déterminer que les soignants tendent à s'effacer dans les observations écrites, pour se concentrer sur des éléments saillants dans la pratique des soignants, alors que dans les réunions, ils inscrivent bien les émotions et traits humains des soignants comme des patients dans leurs discours. Ces différences, qui s'inscrivent dans des constructions particulières, permettent néanmoins de cibler les informations importantes à mutualiser, selon les objectifs des deux modalités de transmission.
\end{abstract}

\begin{abstract}
Nurses' handovers in psychiatry are important moments of exchange in the practice of healthcare professionals. These situations of information sharing are coupled with the existence of written notes, thus creating two modalities of information transmission. This paper proposes to establish, with the tools of macrosyntax and conversation analysis, structures supporting the discourse of carers. Based on the analysis of written and oral statements concerning one patient, we discuss syntactic, lexical and pragmatic phenomena that allow us to distinguish the objectives of the two modes of mutualisation, as well as their realisation. Then we analyse a phenomenon that is transversal to both written and oral productions, reported speech, through a written observation corresponding to the information shared during a handover. We can determine that the carers tend to withdraw in the written observations, in order to concentrate on the salient elements in the professionals' practice, whereas in the meetings, they do share the emotions and human traits of the carers as well as those of the patients in their discourse. These differences, and the structure in which they emerge, make it possible to target the important information to be shared, relative to the objectives of the oral and written productions.
\end{abstract}




\section{Introduction}

Les relèves infirmières en psychiatrie sont, dans la pratique soignante, des moments clé qui sont légiférés et rythment le quotidien des équipes. Ils permettent d'établir des parcours de soins évolutifs et cohérents pour les patients.

Lors de ces temps d'échanges que sont les réunions de relèves infirmières, les informations du dossier patient sont mutualisées. En effet, les représentants des différents métiers relatifs au soin (médecins, infirmiers, aide-soignants, psychologues...) y produisent et partagent leurs savoir institutionnels, des connaissances d'ordre professionnel (un médecin ne dispose pas des mêmes connaissances qu'un infirmier), ou bien des connaissances issues de la vie de la structure, puisque, à travers le contact quotidien, les soignants acquièrent une forme de savoir de façon dynamique sur l'état pathologique des patients.

Les relèves sont des moments profondément langagiers; par conséquent, il nous paraît pertinent de mobiliser des outils et théories des sciences du langage afin d'observer, et de décrire les différentes manières de présenter les informations au sein des réunions de mutualisation. Nous utilisons, pour cet article, le corpus du projet CIPSY, et nous nous sommes concentrés sur les relèves concernant une patiente, sur une semaine. Nous avons considéré à la fois les relèves orales, enregistrées puis transcrites en suivant les conventions ICOR (2013), et les notes du dossier patient, écrites simultanément par l'un ou les mêmes soignants qui suivent les patients concernés par la relève orale.

À partir de ce corpus que nous avons constitué, nous tenterons de voir, à travers les modalités orales et écrites, comment, dans ce contexte particulier, les informations sont mutualisées. Nous montrerons aussi comment celles-ci sont sélectionnées, mises en valeur par le locuteur, pour les rendre plus saillantes. Chaque soignant, dès lors qu'il mutualise des informations auprès des équipes, doit contribuer à la fois à la mémorisation par l'auditoire des informations (ou à défaut, de certaines informations), ainsi qu'à la progression de l'interaction, c'est à dire au bon fonctionnement de la relève.

Nous porterons un regard linguistique au plan qualitatif, sur les structures syntaxicopragmatiques de l'information dispensée par les professionnels du soin au cours de ces relèves. C'est au prisme des théories et outils de l'École Aixoise et du GARS, dans la lignée directe des travaux de Claire Blanche-Benveniste que nous examinerons plus particulièrement deux exemples de structures syntaxiques privilégiées dans les relèves, et deux phénomènes spécifiques, le discours rapporté et les répétitions/reformulations.

\section{Analyses qualitatives : I'information et ses structures}

\subsection{Types d'informations distinctives entre l'écrit et l'oral}

Tout d'abord, nous abordons dans cette partie quelles informations saillantes se retrouvent dans les relèves. Nous analysons les relèves au sujet d'une patiente qui est présente dans l'unité pour une dépression chronique et persistante. En raison de l'inefficacité des traitements, sa prescription a été modifiée de façon très récente, antérieurement à nos premiers enregistrements. Elle fait également face à des problématiques judiciaires, et professionnelles. Du point de vue sémantique, nous pouvons établir le tableau suivant dans lequel sont référencés tous les types d'informations mentionnés et/ou expliqués dans les différentes réunions. En noir, sont notées les relèves orales, en bleu, les notes écrites. La nomenclature suit la logique suivante : les transmissions écrites démarrent par un "E", suivi du numéro de la semaine correspondant à l'enregistrement. L'heure de réunion ou de saisie de note est précisée après un underscore. 
Tableau 1. Contenu sémantique des informations traitées dans les relèves concernant la patiente

\begin{tabular}{|c|c|c|c|}
\hline & & Mentions & Explications \\
\hline \multirow{3}{*}{$\begin{array}{l}\text { Informations } \\
\text { internes }\end{array}$} & État émotionnel & Toutes les relèves & $\begin{array}{c}\text { S1_6h } \\
\text { S1_9h } \\
\text { S1_14h }\end{array}$ \\
\hline & $\begin{array}{l}\text { Qualité de } \\
\text { sommeil }\end{array}$ & $\begin{array}{l}\text { S1_6h } \\
\text { S1_9h } \\
\text { S1-14 }\end{array}$ & $\begin{array}{l}\text { S1_6h } \\
\text { S1_9h }\end{array}$ \\
\hline & $\begin{array}{l}\text { Modifications } \\
\text { prescriptions }\end{array}$ & $\begin{array}{c}\text { S1_9h } \\
\text { S1_14h } \\
\text { S1_21h } \\
\end{array}$ & S1_9h \\
\hline \multirow{3}{*}{$\begin{array}{c}\text { Informations } \\
\text { externes }\end{array}$} & $\begin{array}{c}\text { Problématiques } \\
\text { judiciaires }\end{array}$ & $\begin{array}{c}\text { S1_9h } \\
\text { S1_14h } \\
\text { S2_9h } \\
\text { ES1_17h16 } \\
\text { ES2_20h44 }\end{array}$ & $\begin{array}{c}\text { S2_9h } \\
\text { ES2_10h44 }\end{array}$ \\
\hline & $\begin{array}{c}\text { Problématiques } \\
\text { de travail }\end{array}$ & $\mathrm{S} 1 \_9 \mathrm{~h}$ & $\mathrm{~S} 1 \_9 \mathrm{~h}$ \\
\hline & Proches & $\begin{array}{c}\text { S1_9h } \\
\text { S1_21h } \\
\text { ES1_17h16 } \\
\text { ES1_22h29 }\end{array}$ & $\begin{array}{c}\text { S1_9h } \\
\text { ES1_22h29 }\end{array}$ \\
\hline
\end{tabular}

Comme nous pouvons le voir ci-dessus, diverses informations sont mutualisées lors des transmissions. On peut distinguer deux catégories principales, l'une relevant des éléments internes à la structure et l'autre des éléments externes. En précisant l'information, on trouve divers contenus, à savoir l'état émotionnel de la patiente, sa qualité de sommeil, les modifications de prescription qui ont pu avoir lieu ; et quant aux informations externes, il s'agit de litiges judiciaires, de problématiques qui concernent son travail, ou encore l'évocation des proches de la patiente. Nous avons également précisé si le contenu relevait d'une mention ou d'une explication, en distinguant bien les éléments seulement signalés de ceux à la fois mentionnés mais aussi débattus ou développés.

En premier lieu, il convient de noter qu'un seul type d'information est présent dans toutes les relèves orales : l'état émotionnel. Cette omniprésence n'est pas surprenante, la patiente souffrant de dépression, l'un des symptômes majeurs de sa pathologie est justement son humeur.

"The common feature of all of these disorders [les troubles dépressifs] is the presence of sad, empty, or irritable mood, accompanied by somatic and cognitive changes that significantly affect the individual's capacity to function" (American Psychiatric Association 2013: 155 ).

Dès lors, il paraît cohérent qu'un intérêt particulier soit porté à son état émotionnel.

Dans ce contexte, nous remarquons aussi, et de façon flagrante, une distinction forte entre le contenu des notes écrites et les relèves orales. En effet, si tous les contenus sémantiques sont représentés lors des réunions, ce n'est pas le cas dans les observations écrites. Au regard des éléments informationnels présentés pour cette patiente, nous pouvons ainsi considérer un premier niveau de distinction des informations transmises par les soignants, selon que ladite transmission est orale ou écrite : les informations internes à la structure seront 
préférentiellement mutualisées lors des réunions de relèves, alors que, dans les observations écrites, ce sont les informations externes à la structure qui sont plutôt présentées, et donc non sujettes à discussion lors des relèves orales.

D'autres éléments distinctifs des deux modalités de transmission sont repérables au niveau des constructions syntaxiques et dans une perspective plus micro analytique, au niveau du contenu sémantico-pragmatique.

\subsection{Considérations propres à l'écrit}

Alors que beaucoup de phénomènes dits «spécifiques de l'oral » apparaissent aussi dans de nombreux types d'écrits ${ }^{1}$, il convient d'emblée de préciser que les phénomènes, repérés pendant les relèves (voir plus loin), plus spécifiques de l'interaction orale, ne se retrouvent pas dans notre corpus de notes écrites concernant la patiente.

Les dossiers écrits s'adressent à l'ensemble des équipes de soin, suivront le patient tout au long de son parcours médical et pourront comme le prévoit la loi être réclamés par ce dernier. S'il y a lieu, le dossier pourrait être remis à la justice, et des agents de la justice ou de la police pourraient être conduits à en prendre connaissance, tout comme des pairs en dehors de la structure d'accueil dans laquelle est constitué le dossier, ou encore le patient lui-même.

En effet, il faut bien évidemment prendre en compte la « cible» du dossier écrit par rapport aux réunions entre pairs, à l'issue desquelles ne subsiste aucune trace.

Les différences de formats et de contenu des informations livrées entre l'oral et l'écrit s'expliquent pour une grande partie de cette manière (même si d'autres facteurs interviennent certainement qui peuvent avoir trait à la formation des soignants, à leur expérience, à la représentation de ce que doit être un « écrit », le fonctionnement interne de la structure, etc.).

L'apport de l'information (syntaxe / lexique / sens) se fait à travers trois aspects principaux : 1) des constructions syntaxiques particulières ; 2) des phénomènes lexicaux ; 3) des phénomènes pragmatiques et sémantiques.

\subsubsection{Des constructions syntaxiques particulières}

Nous pouvons observer dans les notes écrites qu'au niveau syntaxique, deux structures particulières sont repérables. Selon l'approche théorique de l'École Aixoise (BlancheBenveniste, 1984, 1990), les unités observées et la méthodologie d'analyse proposée permettent de considérer une syntaxe en deux modules qui se superposent : la microsyntaxe concerne l'organisation des éléments liés grammaticalement, directement ou indirectement, au verbe (les critères de rection fonctionnent) ; la macrosyntaxe décrit l'organisation des énoncés en unités structurantes (prénoyau, postnoyau, innoyau), celles-ci étant reliées discursivement (parfois microsyntaxiquement aussi) à une unité de communication autonome, le noyau.

Suivant ce courant, nous pouvons analyser, d'une part, des phrases (noyaux courts), correspondant à des structures syntaxiques raccourcies. C'est par exemple le cas dans "semble effectivement éteinte ".

D'autre part, nous relevons des phrases avec propositions coordonnées et imbriquées (noyaux complexes) composées de subordonnées, complétives, relatives, constructions

\footnotetext{
${ }^{1}$ Qu'il s'agisse de la poésie, des paroles de chansons mais aussi de la représentation de l'oral dans les dialogues de cinéma, de théâtre, de roman, ou des écrits du numérique : courriels, chats, forums, réseaux sociaux, textos...Et dans la littérature aussi !
} 
prépositionnelles très formelles, comme par exemple « Semble entendre que le soin ne passe pas forcément par l'hyperactivité mais pense néanmoins que sa fille apprécierait de pouvoir aller au centre sportif où elle ne peut a priori pour l'instant pas s'inscrire du fait qu'elle ne peut pas sortir seule dans le parc ».

Bien que le noyau court soit fréquent à l'oral, et qu'on retrouve aussi beaucoup de constructions syntaxiques complexes dans de nombreux corpus d'oraux, ces deux types de structures sont ici propres à l'écrit des soignants : on ne les retrouvera pas dans leurs interactions orales.

\subsubsection{Des phénomènes lexicaux}

Au niveau lexical, les soignants écrivent des notes dans le dossier patient, en utilisant un vocabulaire technique et une terminologie opaque. C'est par exemple le cas pour «baisse thymique " (ce qui veut dire «tristesse »). Nous pouvons aussi trouver des mentions de "apyrétique », dans des relèves concernant d'autres patients, ce qui signifie que la température corporelle est normale, ou encore «TTT» (abréviation pour « traitement ») dans presque toutes les relèves écrites, qui n'est pas en tant que tel du lexique technique, mais relève d'une terminologie non accessible aux non connaisseurs.

Si ces choix lexicaux se retrouvent également à l'oral, on les relève dans une bien moindre mesure.

Ici, il s'agit plutôt d'un vocabulaire préférentiel de l'écrit : en effet, dans les réunions de relèves infirmières, nous trouvons plutôt des périphrases très explicatives pour donner des informations sur la patiente - réalité évoquée plutôt par un lexique opaque dans les notes écrites des soignants.

\subsubsection{Des phénomènes sémantiques et pragmatiques}

Le contenu de l'information mutualisée par le biais des écrits porte sur les faits, les actions des soignants et non sur leurs ressentis ou ceux des patients. Ainsi, peu de notes sont saisies, puisqu'elles dépendent d'éléments saillants dans la pratique du soin. Nous pouvons par exemple voir dans la note écrite suivante que les informations qui y sont mutualisées concernent des aspects pratiques dans la profession des soignants.

\section{Extrait 1 : ES2_20h44}

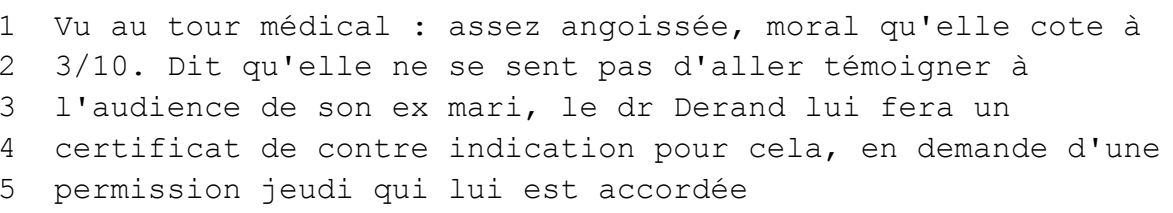

Dans cet extrait, nous pouvons observer plusieurs mentions d'actions réalisées par les soignants : le tour médical (ligne 1), la cotation de l'état dépressif (ligne 1), le fait que le psychiatre doive produire un certificat (lignes 3-4), ainsi que l'accord quant à la demande de permission de la patiente (lignes 4-5). L'aspect majoritaire dans cette observation écrite est l'activité des soignants autour de la patiente, plutôt que la patiente en elle-même. Si le moral est mentionné, il l'est au travers d'une action des soignants, et est abordé par le biais de deux actions de soins : la cotation et le tour médical. 


\subsection{Considérations propres à l'oral}

En ce qui concerne les relèves orales, en suivant le même déroulé que pour les notes écrites, nous pouvons trouver des particularités.

\subsubsection{Au niveau sémantique et pragmatique}

Contrairement aux écrits, le contenu de l'information concerne majoritairement l'expression du ressenti (qu'il s'agisse de celui des patients, comme dans le cas de madame Delattre ou des soignants dans le reste de notre corpus). On peut cependant y trouver des faits ou des actions, mais dans une mesure moindre vis-à-vis de l'autre modalité de transmission. Par exemple, l'extrait suivant concerne la patiente, et est produit dans une relève juste après la saisie informatique de la note que nous venons de voir.

\section{Extrait 2 : Delattre S2_21h}

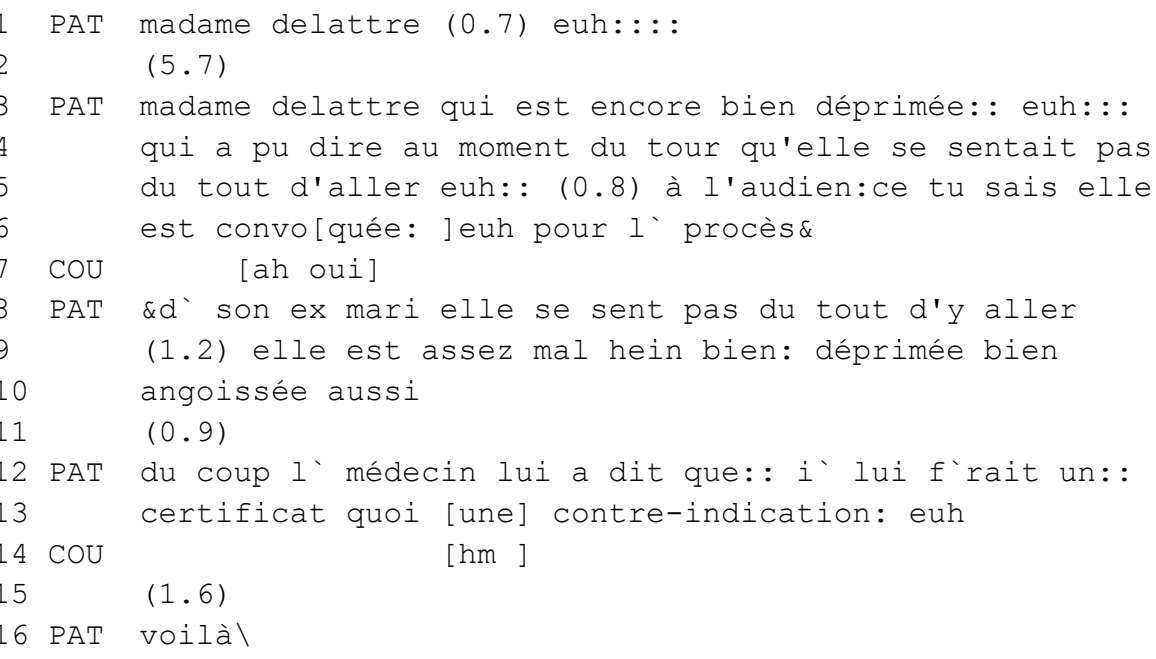

Les informations mutualisées sont globalement les mêmes : PAT présente dans cet extrait l'état émotionnel de la patiente, le fait que le psychiatre doive lui faire un certificat et l'impossibilité manifestée par madame Delattre de témoigner à l'audience de son ex-mari. Cependant, en regardant dans les détails de la conversation, nous pouvons voir que l'accent est ici mis sur le ressenti de la patiente. En effet, après avoir ouvert la séquence concernant la patiente, PAT aborde tout de suite son état en utilisant des marqueurs soulignant le caractère à la fois pérenne et fort de sa dépression : "qui est encore bien déprimée », ligne 3, " elle se sentait pas du tout d'aller » lignes 4-5, répété ligne 8, " elle est assez mal bien déprimée bien angoissée » ligne 9. Par ailleurs, le lien entre l'état émotionnel de la patiente et les réactions des soignants est ici manifeste; comme l'indique « du coup » ligne 12, qui lie syntaxiquement les propositions, et associe la cause (en l'occurrence l'état de la patiente) à la conséquence (la production par le psychiatre d'un certificat).

\subsubsection{Au niveau lexical}

A l'inverse des observations écrites, les relèves orales sont assez transparentes au niveau lexical. Nous pouvons y voir de nombreuses périphrases explicatives des symptômes, plutôt que l'utilisation d'un vocabulaire technique. Bien entendu, ce vocabulaire existe aussi à l'oral, mais dans une moindre mesure : là où les observations écrites sont exclusivement 
opaques dans le choix des mots, l'oral alterne, avec une tendance à utiliser des champs lexicaux plus accessibles à tous. Une explication possible à ce phénomène est le récepteur desdites transmissions : les notes écrites sont adressées principalement aux soignants médicaux et aux infirmiers, alors que dans les réunions, une pluralité de profils est présente, allant de l'agent de service hospitalier, au psychiatre, en passant par le psychomotricien et l'assistant social. Dès lors, puisque la connaissance technique n'est pas systématiquement partagée par tous, le vocabulaire change.

\subsubsection{Au niveau syntaxique}

Toujours dans la veine de l'École Aixoise, nous pouvons repérer une grande fréquence d'utilisation de deux types de constructions macrosyntaxiques :

D'une part nous trouvons beaucoup d'occurrences de prénoyau large + noyau. En utilisant ces structures, très fréquentes dans les interactions orales de la vie quotidienne (BlancheBenveniste, 1990 ; Sabio, 2017), les locuteurs présentent avec plus de force l'information principale par une unité de communication autonome (noyau), préparée longuement dans le discours (prénoyau large ou prénoyaux successifs) avant d'être livrée.

Nous pouvons observer ces phénomènes dans les exemples suivants, provenant de la relève orale se produisant lors de la première semaine d'enregistrement, à la réunion de 9 heures, et concernant madame Delattre :

\section{Extrait 3 : seuls énoncés de LAR}

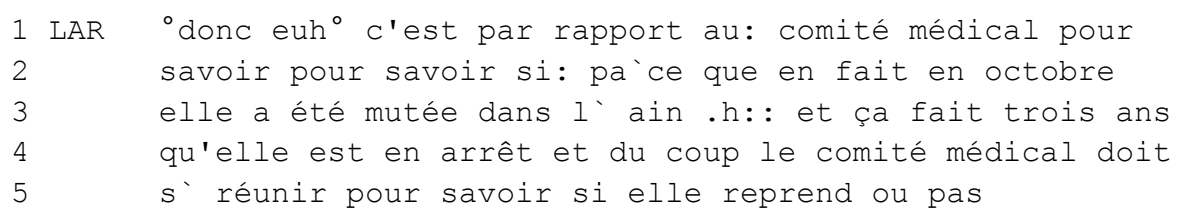

Ici, LAR veut informer ses collègues que le comité médical doit se réunir pour statuer sur la reprise professionnelle éventuelle de la patiente. Elle donne des informations sur sa situation :

- Il faut qu'il la revoie

- En octobre elle a été mutée dans l'Ain

- Elle est en arrêt depuis trois ans

Ces successions de contenus constituent des prénoyaux et une parenthèse, et préparent l'énonciation du noyau ligne 5, pour savoir si (avec reprise des premiers pour savoir si) la patiente reprend son activité ou non.

\section{Extrait 4 : seuls énoncés de IVA}

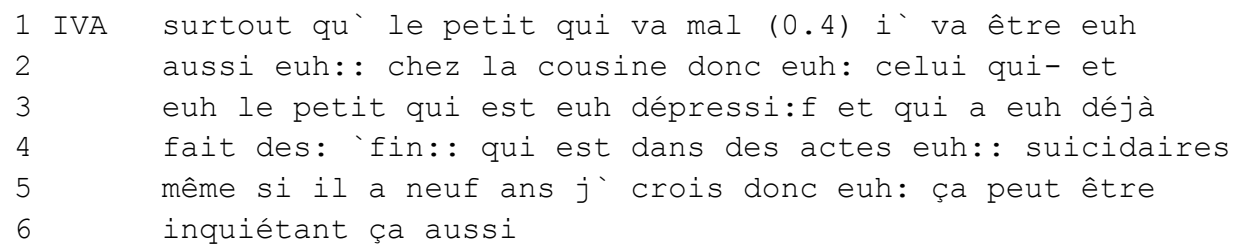

Dans cet exemple, IVA explique ce qui « peut être inquiétant aussi » pour la patiente, pour ce qui concerne ses enfants : IVA donne plusieurs informations sur le plus jeune des enfants (malgré les sept autres interlocuteurs, LAR, GIL, POM, PAT, CHA, LOC, GAL, qui parlent en même temps, d'ailleurs pour enrichir son propos) et continue une même ligne directrice :

- Le petit va mal (ligne 1)

- Il est chez la cousine (lignes 1-2) 
- Il est dépressif (ligne 3)

- Il a déjà eu des gestes suicidaires (ligne 4)

- Il a neuf ans (ligne 5)

Toutes ces informations sont présentées sous la forme de prénoyaux, et alimentent le sentiment d'angoisse de la patiente, qui constitue le noyau « ça peut être inquiétant ça aussi », lignes 5-6.

D'autre part, nous avons pu observer, à de nombreuses reprises, des noyaux successifs liés discursivement (Blanche-Benveniste, 1990, 1997), parfois à analyser comme reformulations. Ces constructions ajoutent une nouvelle information qui est intéressante à développer (qu'il s'agisse d'une explication, d'une forme d'argumentation, d'exemplification).

Par exemple, nous trouvons :

\section{Extrait 5 : noyaux successifs}

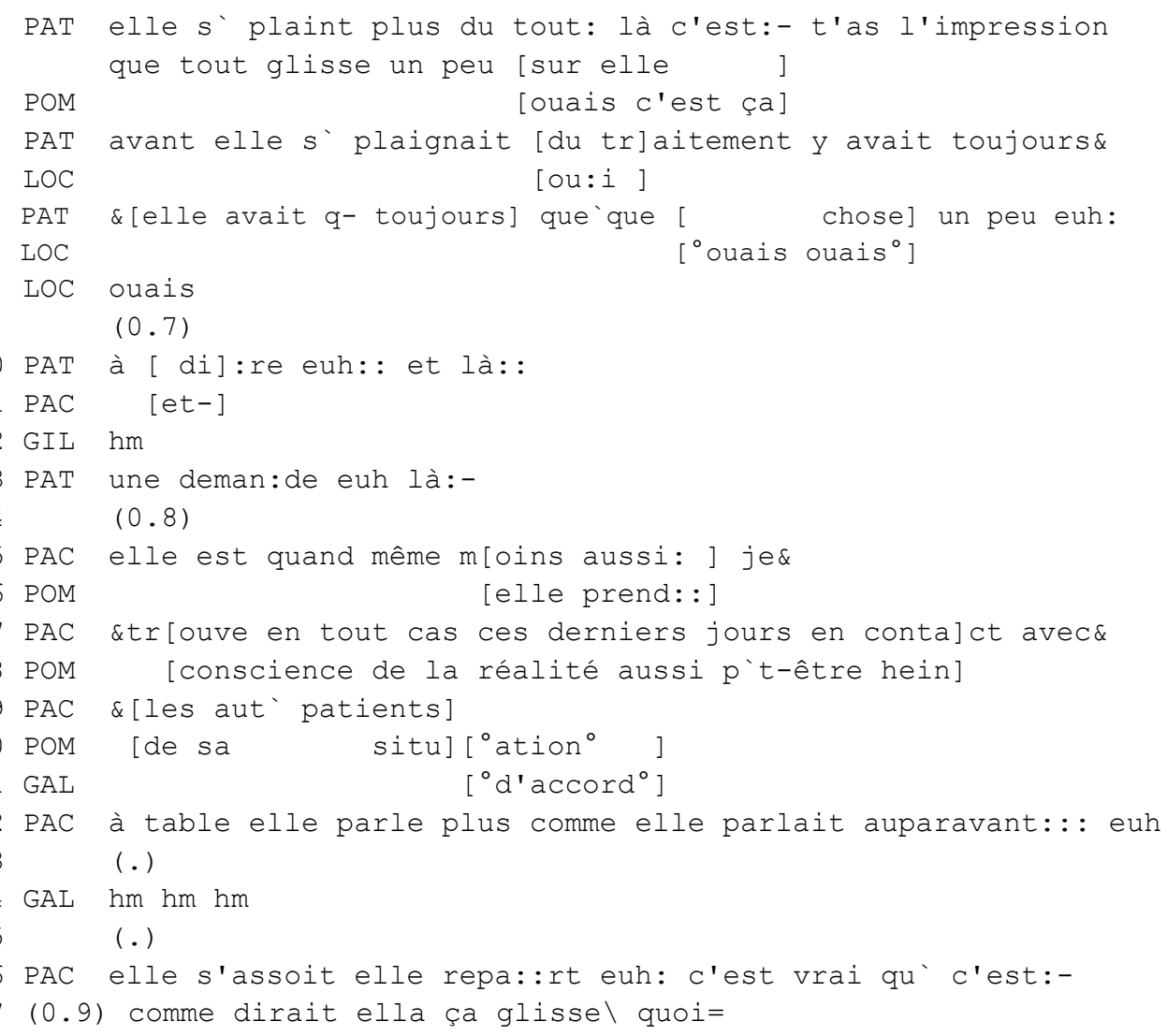

À partir de ces observations syntaxiques, nous pouvons décrire comment les soignants rendent saillante l'information qu'ils veulent souligner, renforcer, pour la faire mémoriser :

- - la structure « prénoyau large » met en exergue et renforce le noyau qui suit ;

- - quant à la succession de noyaux liés discursivement et souvent collaboratifs, chaque locuteur ajoute un nouveau noyau pour enrichir la même information. Ici, le message est que la patiente a changé et que « tout glisse sur elle ». Ces types de structures permettent d'expliciter, de modifier, de justifier, de renforcer l'information à mémoriser. 
Nous avons également pu justement observer des phénomènes langagiers souvent consubstantiels à ces constructions au niveau syntaxique au sein des relèves orales, comme les reformulations et les répétitions. Il s'agit de reprises à l'identique ou avec des expansions, exemplifications, explicitations d'un contenu pré-énoncé. Pour re-dire le même message, la construction syntaxique sera modifiée, tout comme le lexique.

Plusieurs catégories peuvent être distinguées dans ces phénomènes, selon qui produit l'énoncé source, et qui le reformule/répète.

Nous différencions ainsi les auto répétitions/auto reformulations (où le locuteur se répète, ou se reprend), des hétéro répétitions/hétéro reformulations (l'interlocuteur reprend une information donnée par le locuteur) (voir par exemple Ursi et al., 2018 ; Schegloff, Jefferson, et Sacks, 1977) :

\section{Extrait 6 : auto reformulation}

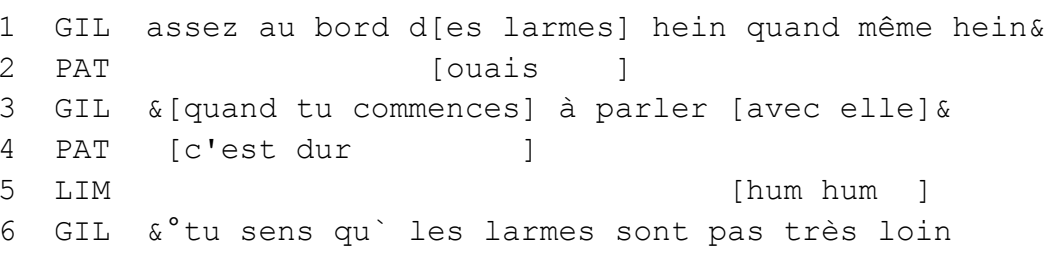

Nous pouvons citer cette auto reformulation «assez au bord des larmes » comme énoncé source, et « hein quand même hein quand tu commences à parler avec elle tu sens que les larmes sont pas très loin » comme énoncé reformulé. Dans cet exemple, une même locutrice produit les deux énoncés, qui varient syntaxiquement et lexicalement. En ajoutant un élément contextuel, la participante insiste sur cette information en étayant son discours, le situant, et l'explicitant.

Nous relevons également des hétéro reformulations, comme c'est le cas dans l'extrait suivant :

\section{Extrait 7 : hétéro reformulation/répétition}

1 IVA elle nous dit $r-e[: l l e$ parle peu: ]

2 GIL [elle nous dit rien hein]

Dans cet extrait, IVA produit un énoncé source qui est repris par GIL sous la forme d'une hétéro-reformulation, en chevauchement avec une auto-reformulation produite par IVA ellemême.

Nous trouvons aussi de nombreuses répétitions, auto et hétéro répétitions :

\section{Extrait 8 : répétitions dans Delattre S1_9h}

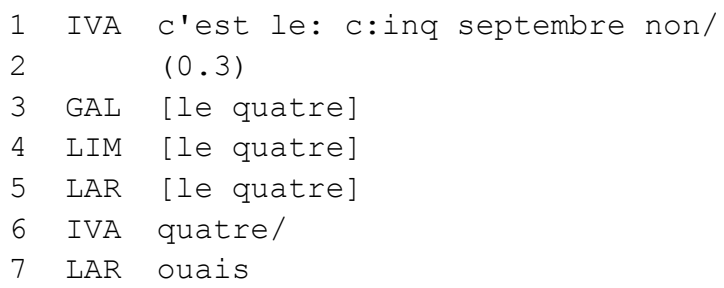

Ici, IVA produit une hétéro-répétition du contenu produit simultannément par GAL, LIM, et LAR. Cet énoncé, dit choral, nous indique également une forme dimension collaborative dans la relève infirmière, ainsi que l'appartenance à un groupe social plus large (Lerner 2002). 
Le phénomène de "locuteur collectif » (Loufrani, 1985) ou d'énoncés / constructions collaboratifs (André, 2010 ; Mondada, 1999 ; Traverso, 2012) se retrouve également dans nos données : le locuteur 2 «termine » l'énoncé suspendu du locuteur 1.

\section{Extrait 9 : énoncé collaboratif dans Delattre S1 9h}

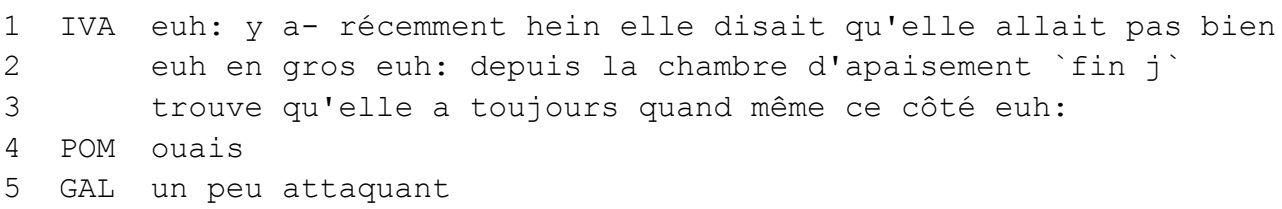

Ici, GAL complète le tour de IVA. Ce faisant, elle montre un alignement avec l'activité en cours, c'est-à-dire qu'elle adopte la même orientation discursive que IVA (Mondada, 1999). Ce phénomène d'alignement, passant par un énoncé collaboratif, nous montre une forte cohésion au sein des relèves entre les soignants.

\section{Une structure transversale aux modalités de transmissions : le discours rapporté}

Le discours rapporté (désormais DR) est une modalité syntaxique de prise en charge, au sein d'un discours donné, d'un discours tiers. De façon typique, il s'agira d'une forme du type " $\mathrm{X}$ a dit $\mathrm{Y} »$, où $\mathrm{X}$ correspond au locuteur ayant formulé le discours en première instance, et Y la reformulation de ce dernier. En linguistique, ce format de production a été étudié selon de nombreux aspects, qu'il s'agisse de ses fonctions au niveau du récit (Holt, 1996; Moreno, 2016), de l'argumentation (Rosier, 2008) ou encore, au sein des relèves, de la dimension collaborative qu'il entraîne (Maritaud, 2020), ainsi que le lien entre la narration et le DR (Bangerter, Mayor, et Doehler, 2011). Nous distinguerons ici le DR direct $(D R D)$, ne présentant pas de variation de déictiques et en temps-aspect-mode, du DR indirect (DRI), comprenant une adaptation de ces derniers au contexte de production. Nous verrons également des formes dites narrativisées $(D R N)$, qui consistent à rapporter l'action de parole sans rapporter les propos. Il s'agit d'un phénomène très utilisé dans ce contexte professionnel. Nous utiliserons ici les outils de l'analyse conversationnelle, développée par Sacks, Schegloff et Jefferson (1974) à partir de l'ethnométhodologie de Garfinkel (1967). Il s'agit d'une discipline visant à étudier le langage comme étant une manifestation d'une situation sociale donnée.

Dans cette partie, nous allons comparer le même contenu informationnel, à l'oral et à l'écrit. Les deux modalités présentent des formes de DR, et nous donnent de nouveaux indices dans les spécificités des relèves orales et des observations écrites. Il s'agit de la même patiente que précédemment, et la soignante prenant la parole à l'oral est également celle qui saisit la note écrite. L'objectif principal des deux relèves est de mutualiser un appel que cette même soignante a reçu de la part de la mère de la patiente dans la journée. Dans les extraits suivants, un code couleur signifiera les occurrences de discours rapporté. L'introduction grammaticale de la séquence (désormais inquit) sera en souligné, et les propos rapportés en gras.

\subsection{Le DR à l'oral}

L'extrait suivant correspond à l'intégralité d'une réunion de relève concernant la patiente. $\mathrm{BER}$, qui mène la réunion, va présenter son actualité clinique, puis entrer dans une forme de récit d'expérience vécue afin de mutualiser son propre vécu. 


\section{Extrait 10 : l'appel de la mère}

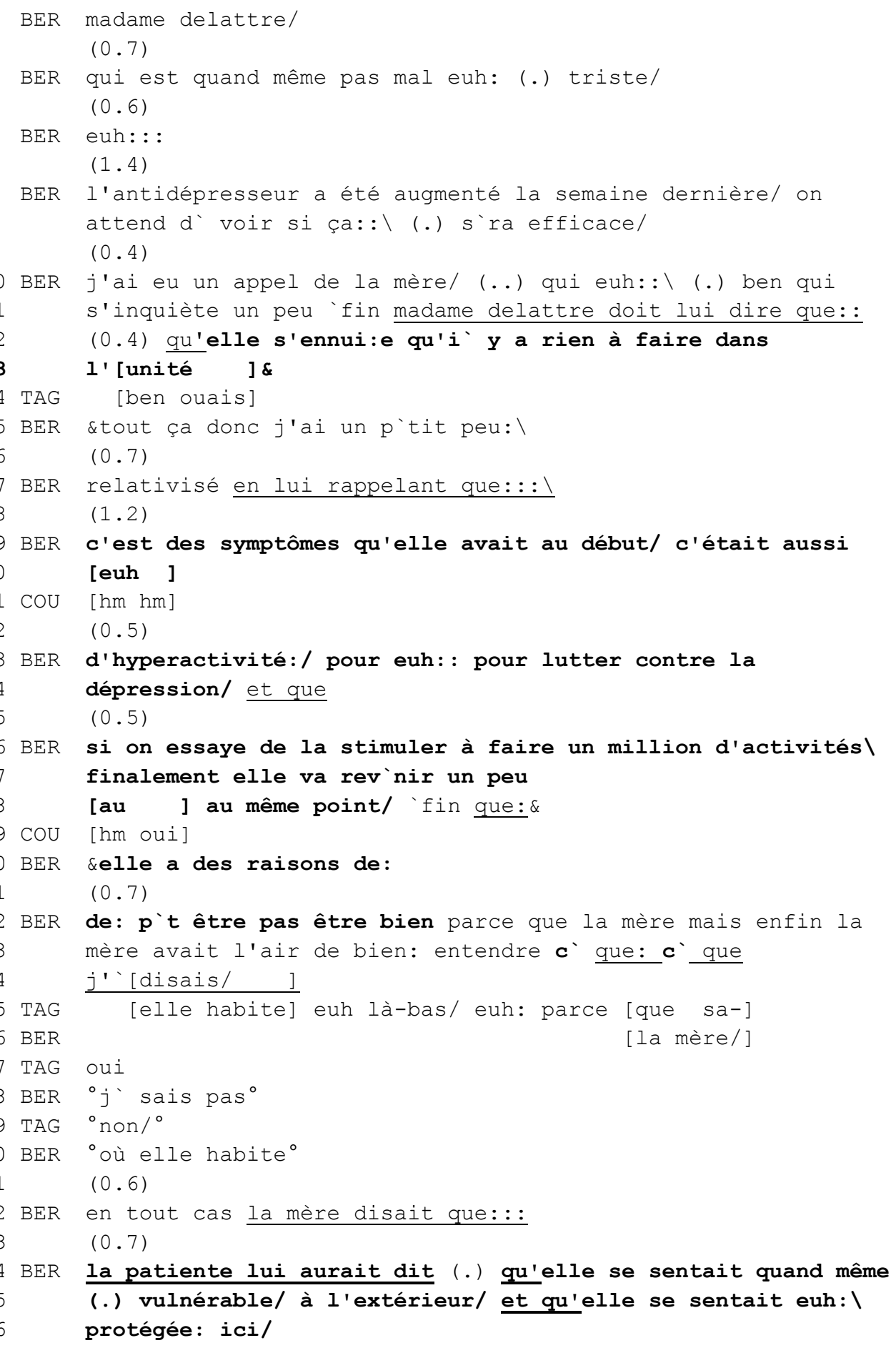




\subsubsection{Considérations informationnelles}

Lignes 1 à 8 , BER présente, en tant que meneuse de la relève, le nom de la patiente en question (ligne 1), puis des informations générales la concernant. Cette structure est commune à toutes les relèves de notre corpus, et est gérée par un participant endossant ce rôle tout au long de la réunion. À partir de la ligne 10, elle entre dans des considérations plus précises en exposant des informations qu'elle a pu avoir directement, et que les autres participants ne connaissent pas encore. Lignes 10 à 13, elle rapporte " un appel de la mère », rendant ainsi audible aux autres participants un élément qu'elle seule a pu recevoir. Dans cet appel, la mère de la patiente elle-même rapporterait les propos de sa fille, témoignant de son ennui (lignes 12-13). Le conditionnel ici tient dans le fait que BER utilise le verbe « devoir» qui fait ici office de modalisateur. Suite à cette première séquence, BER poursuit en précisant ses réponses face au discours tenu lors de l'appel téléphonique (lignes 17 à 32). Mutualiser une telle réponse est très importante dans la cohésion d'équipe : en effet, en apportant des éléments de réponses à ses collègues, BER leur permet d'avoir un propos cohérent avec le sien face aux proches de la patiente en question. De la même façon, elle permet aux participants à cette réunion de réagir face au discours qu'elle a pu tenir.

Lignes 42 à 46, le double DR imbriqué (BER citant la mère, qui elle-même cite la patiente) permet à l'infirmière de donner la responsabilité de l'appréciation de l'état de madame Delattre et des propos rapportés à la mère : en explicitant ce double enjeu de représentation des propos, elle négocie la véridiction des propos. On le voit par ailleurs dans le choix du temps verbal ligne 44 : " la patiente lui aurait dit ». En employant le conditionnel passé, BER accentue l'aspect hypothétique de l'appréciation de la mère face à sa fille, tout comme les propos qui sont attribués à la patiente lors de la conversation téléphonique.

Cet extrait, riche en séquences de DR, nous montre donc plusieurs aspects importants dans son usage lors des relèves. D'une part, il permet d'introduire et d'étayer des séquences informationnelles relatives au vécu personnel de la soignante, contrairement à l'actualité clinique partagée par tous, présente dans les premières lignes de la transcription. En utilisant ce format de production, BER rapporte également ses propres propos. Ce faisant, elle permet d'établir une forte cohérence des discours de l'équipe de soins face aux proches des patients, et met en perspective son activité individuelle au regard des autres soignants. Quant aux séquences de DR attribuées à la mère de la patiente, elles permettent à la fois de rendre disponibles les propos du proche à l'ensemble des soignants, mais aussi à apporter des éléments sur l'état psychique de la patiente, selon l'avis des personnes qui la connaissent le mieux. Le DR, dans les relèves orales, revêt donc un intérêt informationnel tout particulier.

\subsubsection{Une forme de récit oral}

Nous pouvons également noter une corrélation très étroite entre DR et récit. Selon Labov (1972), un récit est caractérisé par au moins deux propositions syntaxiques qui suivent un déroulement temporel logique. Dans notre cas, on retrouve effectivement cette succession, à partir de la ligne 10, correspondant aux premières séquences de DR. Le tableau ci-dessous présente la correspondance entre les propositions syntaxiques et les informations mutualisées : 
Tableau 2. Chronologie du récit dans la relève orale au regard du DR

\begin{tabular}{|c|c|c|c|c|}
\hline $\begin{array}{c}\text { Ordre dans } \\
\text { l'extrait }\end{array}$ & Élément 1 & Élément 2 & Élément 3 & Élément 4 \\
\hline $\begin{array}{c}\text { Ordre dans } \\
\text { la narration }\end{array}$ & 2 & 1 & 3 & Inconnu \\
\hline $\begin{array}{c}\text { Numéros de } \\
\text { lignes }\end{array}$ & Ligne 10 & Lignes 11-13 & Lignes 17-32 & Lignes 42-46 \\
\hline $\begin{array}{c}\text { Contenu } \\
\text { relatif au DR }\end{array}$ & $\begin{array}{c}1 \text { séquence de } \\
\text { DRN }\end{array}$ & $\begin{array}{c}1 \text { séquence de } \\
\text { DRI }\end{array}$ & $\begin{array}{c}\text { 1 séquence de DRD, } \\
\text { 2 inquits en incise, } \\
\text { reprise anaphorique }\end{array}$ & $\begin{array}{c}\text { 1 séquence de } \\
\text { 2 inquits en incise }\end{array}$ \\
\hline $\begin{array}{c}\text { Information } \\
\text { globale }\end{array}$ & $\begin{array}{c}\text { Appel de la } \\
\text { mère }\end{array}$ & $\begin{array}{c}\text { 1er DR } \\
\text { potentiel de la } \\
\text { patiente par la } \\
\text { mère }\end{array}$ & $\begin{array}{c}\text { Réponse de la } \\
\text { soignante }\end{array}$ & $\begin{array}{c}\text { 2ème DR de la } \\
\text { patiente par la } \\
\text { mère }\end{array}$ \\
\hline
\end{tabular}

Il est particulièrement intéressant de constater que les quatre éléments sont ponctués par une séquence de DR. Il s'agit même de son constituant exclusif pour le premier, le troisième et le quatrième. Nous pouvons observer que dans le deuxième, une incise est faite lignes 32-34, en donnant à voir aux participants de la relève la réaction de la mère au discours qu'elle rapporte.

Ochs (2007) a étudié le fait, au sein de sa narration, de stipuler la réaction d'une tierce personne à l'action rapportée. Elle considère en effet que la narration, en plus d'établir une succession d'événements, présente souvent une situation dite problématique : "the life events that receive narrative attention tend to be cast as unusual, in that they are unexpected or problematic" (ibid. : 271). Dans notre cas, la situation problématique peut être l'appel de la mère, qui trouve sa résolution dans le fait qu'elle " avait l'air de bien entendre », ligne 33.

La production d'un récit entraîne des conséquences sur le plan interactionnel :
"narrating personal experience allows us to reconcile how we (and others) behaved in the past and how we project ourselves (and others) in an as-yet-unrealized future with current self- understanding" (ibid. : 285)

En explicitant ses dires et ses actions sous la forme d'un récit ponctué de DR, BER construit donc sa propre identité, en adéquation avec les actions qu'elle a pu avoir, mais permet aux autres participants de reproduire son comportement, ses propos et ses actions, que ce soit dans un cadre de réunion, de saisie d'observation ou encore face à la patiente et sa famille. Ainsi, elle participe également à la construction d'une identité collective d'équipe.

L'autocitation que BER produit lignes 17-32 est par ailleurs sous la forme de DRD, dont l'usage dans les relèves infirmières a déjà été analysé comme un élément constitutif d'une forme de de culture professionnelle :
"[the] use of DRS is instrumental in the enactment and sharing of a professional culture that involves dealing with and solving technical problems, as well as displaying and sharing relevant experience" (Bangerter, Mayor, et Doehler 2011 : 18)

La relève orale, si elle présente des informations, consiste ici aussi en un récit de vécu personnel de la soignante entrecoupé d'éléments plus proches de l'activité du soin, et de l'équipe en tant que telle, ce qui permet de créer une forte cohésion à la fois dans les savoirs mutualisés et dans les pratiques professionnelles. Mais qu'en est-il des observations écrites? 


\subsection{Le DR à l'écrit}

Le prochain extrait constitue une note saisie par la même infirmière, BER, dans le dossier patient, une heure après la relève orale que nous venons d'étudier. Elle y expose l'appel de la mère, en utilisant une nouvelle fois du DR.

\section{Extrait 11 : relève écrite correspondant à la transmission orale}

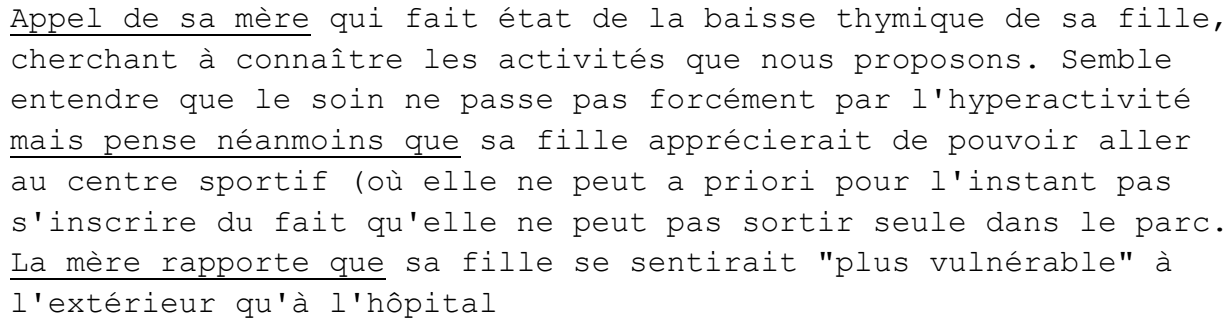

Dans cette observation, nous remarquons trois séquences de DR. La première, lignes 1-2, pose le cadre d'interaction qui est rapporté "appel de sa mère », et précise de façon simultanée l'observation du parent de la patiente et de sa demande, "cherchant à connaître les activités que nous proposons » constituant une proposition participiale. Herslund (2000: 87) nous indique en effet que « le PP [participe présent] désigne avec son verbe principal une seule situation », rendant ainsi syntaxiquement une seule temporalité. Dans la suite de l'extrait, BER ne s'inscrit dans son écrit que de façon implicite : par exemple ligne 3 , la mère "semble entendre», ce qui implique le discours préalable de la soignante, mais nous n'observons pas de manifestation directe de sa présence dans son récit. Ligne 4, BER utilise la conjonction « mais », qui nous montre cette fois une rupture entre les deux propositions coordonnées, que nous considérons donc comme deux éléments distincts, qui ne relèvent donc pas de la même situation. Dans cette proposition, elle produit une deuxième séquence de DR (lignes 4-5), qui relève une nouvelle fois du DRI. Il s'agit par ailleurs d'une information qui n'a pas été mentionnée au préalable dans la relève orale. Enfin, elle produit un dernier DR lignes 7-8, dans lequel elle cite la mère de la patiente, mais ne précise pas que le discours du parent viendrait originellement de sa fille.

Plusieurs différences sont notables entre les deux formats de productions. Tout d'abord, comme nous avions pu le constater dans le tableau des types d'informations de la première partie, il n'y a pas à l'écrit de mention de l'actualité clinique de la patiente ; celle-ci est mutualisée lors des relèves orales et entre alors dans le savoir commun de tous les soignants.

En se concentrant sur les séquences de discours rapporté, nous pouvons constater que ces modalités sont manifestées de façon plus saillante à l'oral. En effet, les verbes du « dire » à l'oral sont plus nombreux (on peut trouver dans les extraits précédents quatre fois le verbe « dire » et une fois le verbe « rappeler » à l'oral, alors qu'à l'écrit une seule forme verbale est présente, « rapporter »). Ces différences verbales entraînent un décrochement énonciatif plus fort, c'est-à-dire une plus forte mise en valeur du tiers dont on rapporte les propos à l'oral qu'à l'écrit. Par extension, on peut également considérer une certaine saillance des propos formulés en première instance : en donnant plus de poids au tiers énonciatif que l'on cite, on donne aussi plus de poids aux propos qu'il a tenus. À l'écrit, la faible quantité de verbes de dire entraîne un décrochement énonciatif plus faible, et met moins en valeur les discours formulés en première instance.

Cette distinction nous informe sur une autre différence entre relève écrite et relève orale : si nous avons pu constater que le décrochement énonciatif était plus fort à l'oral, c'est parce que la présence du locuteur dans ses propos est également plus saillante dans cette modalité de transmissions d'informations. En effet, comme nous avons pu le voir, à l'oral, les 
soignants donnent davantage leur avis, ils racontent de façon plus visible ce qu'ils ont personnellement vécu. Ce constat est notamment repérable au travers de l'utilisation des pronoms personnels et de leurs référents : à l'oral, il y aura beaucoup plus de " je » qui correspondent aux soignants qu'à l'écrit, que ce soit dans les données concernant madame Delattre ou dans le reste du corpus. Au travers du lexique employé, nous pouvons également remarquer que la relève écrite est plus technique que la relève orale : pour une même information, nous trouvons « baisse thymique » à l'écrit, versus « bien triste ", " s'ennuie » à l'oral. Enfin, dans un souci d'exhaustivité, la soignante a également mentionné le centre sportif de l'unité et des sorties autorisées au sein de l'hôpital, à l'écrit seulement, probablement parce qu'il appartient au savoir collectif des soignants qu'il n'est pas possible à l'heure actuelle pour la patiente d'en profiter.

\section{Conclusion}

Les modalités de transmissions orales et écrites offrent donc des objectifs distincts, et des réalisations différentes dans la présentation d'une même information. Nous constatons que lors des relèves écrites, le soignant a tendance à s'effacer au profit d'une information plus technique, plus descriptive et sur laquelle il a moins de pouvoir, alors que les relèves orales permettent une forte intégration du soignant en tant que personne, au travers de son expertise, de son vécu et plus globalement des événements qui ont pu se produire au sein de l'unité.

La parole en psychiatrie, elle-même marquée par le soin relationnel, nous permet donc de voir que l'écoute des soignants est prioritaire, selon deux temporalités distinctes. D'une part, les soignants, pour pouvoir rapporter les propos entendus, doivent y être attentifs. D'autre part, lors des relèves, l'écoute active de l'équipe permet la mémorisation des informations dans l'objectif du suivi du patient, de la collaboration avec le médecin, les proches, la justice ou encore la structure médicale. Le DR ouvre également à la notion de vérité, la valeur de vérité, et renforce ou valide la crédibilité de la parole du soignant, tout comme celle du patient ou de ses proches. Cette étude de cas et ses résultats montrent que seule une grande capacité d'écoute permet cette très grande fréquence de DR : sans écoute, pas de DR. Le DR renforce / valide la crédibilité de la parole du soignant, en permettant la mémorisation des informations cruciales pour le suivi du patient.

Dans un cadre interactionnel articulé aux approches syntaxiques (syntaxe élargie de l'École Aixoise) :

- Le phénomène de discours rapporté

- Les constructions noyaux mises en avant, préparées longuement, ou associées à d'autres noyaux associés pour se renforcer mutuellement

- Les répétitions, reformulations, et plus globalement les phénomènes de reprises incessantes du soignant livrant la parole et/ou le comportement du patient

permettent la progression de l'interaction, selon des aspects de la temporalité, de séquentialité. En effet, ces faits langagiers soulignent, pointent, appuient certaines informations ainsi choisies, sélectionnées et donc mises en exergue, renforcées, et par extension mémorisées.

\section{Références}

American Psychiatric Association (éd.). (2013). Diagnostic and statistical manual of mental disorders: DSM-5, Washington, D.C.: American Psychiatric Association. 
André Virginie. (2010). Éléments de construction collaborative du discours au sein de réunions de travail : la reprise et le couple oui non. Pratiques, vol. 147-148, p.199222.

Bangerter Adrian, Mayor Éric, Pekarek-Doehler Simona. (2011). Reported Speech in Conversational Storytelling During Nursing Shift Handover Meetings. Discourse Processes, vol. 48, n³, p. 183-214.

Benzitoun Christophe, Dister Anne, Gerdes Kim, Kahane Sylvain, Pietrandrea Paola, Sabio Frédéric. (2010). Tu veux couper là faut dire pourquoi. Propositions pour une segmentation syntaxique du français parlé, Actes du Congrès Mondial de Linguistique Française (CMLF 2010), p. 2075-2090.

Berrendonner Alain. (2002). Les deux syntaxes. Verbum, vol. 24, p. 23-35.

Blanche-Benveniste Claire, Bilger Mireille, Rouget Christine, van den Eynde Karel. (1990). Le français parlé. Études grammaticales Sciences du langage. Paris: Éditions du CNRS.

Blanche-Benveniste Claire, Deulofeu José, Stefanini Jean, van den Eynde Karel. (1984). Pronom et syntaxe. L'approche pronominale et son application au français. Paris: SELAF.

Blanche-Benveniste Claire. (1987). Syntaxe, choix de lexique et lieu de bafouillage. D.R.L.A.V. Documentation et Recherche en Linguistique Allemande Vincennes, vol. 36-37, p. 123-157.

Blanche-Benveniste Claire. (1990). Grammaire première et grammaire seconde : l'exemple de EN. Recherches sur le français parlé, vol. 10, p. 51-73.

Blanche-Benveniste Claire. (1997). Approches de la langue parlée en français. Gap, Paris : Ophrys - L'Essentiel.

Blanche-Benveniste Claire. (2000). Phrase et construction verbale. In M. Charolles, P. Le Goffic, M.-A. Morel, (éds.), Y a-t-il une syntaxe au-delà de la phrase ? Verbum, T. XXIV, ${ }^{\circ} 1-2$. Nancy: Presses universitaires de Nancy, p. 37-55.

Blanche-Benveniste Claire. (2003). Le recouvrement de la syntaxe et de la macrosyntaxe. In A. Scarano, Macro-syntaxe et pragmatique. L'analyse linguistique de l'oral, Roma: Bulzoni, p. 53-75.

Delomier Dominique et Morel Mary-Annick. (2002). Les deux voix de l'énonciateur en français oral spontané (propriétés intonatives). Faits de Langues, n 19, p. 221-230.

Garfinkel Harold. (1967). Studies in ethnomethodology. Englewood Cliffs: Prentice-Hall

Groupe ICOR. (2013). Conventions ICOR. [http://icar.univ- lyon2.fr/projets/corinte/documents/2013_Conv_ICOR_250313.pdf]

Herslund Michael. (2000). Le participe présent comme co-verbe. Langue française, vol. 12, $\mathrm{n}^{\circ} 1$, p. 86-94.

Holt Elizabeth. (1996). Reporting on Talk: The Use of Direct Reported Speech in Conversation. Research on Language and Social Interaction, vol. 29, n³, p. 219245.

Lerner Gene H. (2002). Turn-Sharing: The Choral Co-Production of Talk-in-Interaction. In C. E. Ford, B. A. Fox, et S. A. Thompson (eds.), The Language of Turn and Sequence. Oxford University Press, p. 225-256.

Loufrani Claude, (1985). Le Locuteur collectif. Typologie de configurations discursives. Gars, Recherches sur le français parlé, vol. 6, p. 169-193. 
Maritaud Louis. (2020). La gestion d'un épisode de crise en psychiatrie : la relève infirmière comme plateforme de décision collaborative. Interstudia, n²8, p. 101-112

Mondada Lorenza. (1999). L'organisation séquentielle des ressources linguistiques dans l'élaboration collective des descriptions. Langage \& société vol. 89, n¹, p. 9-36.

Moreno Anaïs. (2016). Le discours rapporté dans les interactions ordinaires : l'effet de la proximité et des communautés de pratique sur sa construction à l'oral et à l'écrit. Thèse en sciences du Langage, Université Paris Ouest Nanterre La Défense.

Ochs Elinor. (2004). Narrative Lessons. In A. Duranti (ed.), A Companion to Linguistic Anthropology. Oxford: Blackwell, p. 269-89.

Rosier Laurence. (2008). Le discours rapporté en français. Paris : Éditions OPHRYS.

Sabio Frédéric, (2017). Quel statut accorder aux faits de " détachement » dans un Modèle syntaxique à deux composantes? Travaux de linguistique, vol. 1, nº74, p. 109-139

Sacks Harvey, Schegloff Emanuel A. and Jefferson Gail. (1974). A Simplest Systematics for the Organization of Turn-Taking for Conversation. Language, vol. 50, nº , p. 696735 .

Schegloff Emanuel A., Jefferson Gail and Sacks Harvey. (1977). The Preference for SelfCorrection in the Organization of Repair in Conversation. Language, vol. 53, n², p. 361-382.

Traverso Véronique. (2012). Analyses interactionnelles : repères, questions saillantes et évolution. Langue française, $\mathrm{n}^{\circ} 175$, p. 3-17.

Traverso Véronique. (2012). Longues séquences dans l'interaction : ordre de l'activité, cadres participatifs et temporalités. Langue française, $\mathrm{n}^{\circ} 175$, p. 53-73.

Ursi Biagio, Oloff Florence, Mondada Lorenza et Traverso Véronique. (2018). Diversité des répétitions et des reformulations dans les interactions orales: défis analytiques et conception d'un outil de détection automatique. Langages, ${ }^{\circ} 212$, p. 87-104. 